\title{
STUDIES ON THE EFFECTS OF TUBERCULIN.
}

\author{
By PETER M. HOLST, \\ formerly Assistant at the Institute. \\ (From the Hygienic Institute of the University of Christiania.)
}

(With 2 Text-figs.)

\section{INVESTIGATIONS CONCERNING THE EFFECTS OF TUBERCULIN IN THE HEALTHY ORGANISM.}

THE problem of the effects of tuberculin ${ }^{1}$ in the organism in many respects is unsolved. To throw some light thereon has been the main object of the experiments here described in this paper.

In his original paper Koch (1912) stated that an injection of 2 c.c. of tuberculin into a healthy guinea-pig to all appearances had no effect. Nevertheless he was of the opinion that tuberculin has toxic effects upon the healthy organism, probably upon the white corpuscles. I shall return to this statement later. Most authors agree that tuberculin has practically no toxic effects upon the normal organism (Behring (1912), Marie and Tiffman (1900), Löwenstein (1908), Ruppel and Rickmann (1910)). Where excessive doses are employed, the toxic effects of the glycerin in the tuberculin will come into consideration.

About the fate of the tuberculin in the organism very little is known. The subject has not frequently been treated in the literature on tuberculin. Slatinéanou and Jonesco-Mihaesti (1908) injected 10 c.c. of tuberculin intravenously into a normal goat. After 12 and 18 days, serum and urine from the goat were injected into tuberculous guinea-pigs. The subsequent rise of temperature in these animals was ascribed to the presence of tuberculin in the goat's blood and urine. Slatinéanou and Danielopol (1908) were, however, never able to demonstrate the presence of tuberculin in the blood of normal guinea-pigs which they had treated with tuberculin, and a similar result was also obtained by Wolff-Eisner (1909). Franceschelli (1913) found that tuberculin was eliminated through the kidneys after 6 hours. By means of the intradermoreaction the tuberculin could be quantitatively recovered in the urine of the animals. Neither by this method nor by that of complementbinding was he able to demonstrate any trace of tuberculin in the blood a few hours after the injection.

To study the fate of tuberculin in the organism more closely I injected it intravenously and intraperitoneally into normal rabbits and guinea-pigs. After a certain interval blood was drawn from the vein or the animal was

\footnotetext{
1 "Tuberculin," throughout this paper refers to Koch's old tuberculin.
} 
killed by bleeding. Serum and peritoneal exudate were injected into the skin of tuberculous animals or used for the Pirquet test. As a test-object for this test I have used my own arm, the skin of which is very sensitive to tuberculin. Controls were made with known dilutions of tuberculin. The results were as follows.

A rabbit (weight 5000 g.) was injected intravenously with $2 \cdot 25$ c.c. of tuberculin. If the total amount of blood is estimated to be about 7 per cent. of the bodyweight, a rabbit of $5000 \mathrm{~g}$. will have about $350 \mathrm{~g}$. of blood, and immediately after the injection of 2.25 c.c. of tuberculin it must be supposed to contain about 0.0064 g. of tuberculin per c.c. and 0.2 c.c. of the blood will contain about 0.0013 g., a quantity, which, when injected intradermally into a tuberculous guinea-pig, will give a typical reaction. In fact this proved to be true. $0 \cdot 2$ c.c. of blood drawn immediately after the injection, gave a typical necrosis after injection into the skin of a tuberculous guinea-pig. This experiment, which was repeated several times, shows that tuberculin is circulating in the blood immediately after its injection into the vascular system.

To find out how long tuberculin remains in the circulation, blood was drawn from the vein $\frac{1}{2}$ and $1 \frac{1}{2}$ hours after the injection. In serum obtained from this blood tuberculin could not be found by the method described above. Even much greater doses of tuberculin disappear almost immediately from the blood. Thus a young rabbit, weighing $900 \mathrm{~g}$., was injected intravenously with 2 c.c. of tuberculin. Death followed after a few minutes. 12 c.c. of blood, supposed to contain about $0.4 \mathrm{~g}$. of tuberculin, were dried with a fan, and dissolved in as little water as possible. $0 \cdot 2$ c.c. of this liquid, which had a volume of 2 c.c. produced no reaction when injected intradermally into a tuberculous guinea-pig. A small quantity of the solution was smeared into a superficial scarification on my arm in the manner of the Pirquet test, also without reaction, whereas a control with tuberculin diluted 1 to 10 with salt solution gave a very strong positive reaction.

While these experiments clearly show that tuberculin rapidly disappears when injected into the blood, it still remained to be seen whether it disappears as rapidly from other parts of the organism. I therefore injected 1 c.c. of tuberculin subcutaneously into the ear, and 2 c.c. intraperitoneally into a young rabbit (weight 900 g.), which was killed after half an hour. From the ear was obtained a liquid with the appearance and smell of tuberculin. The peritoneal cavity contained about 15 c.c. of a yellowish, cloudy fluid which did not smell of tuberculin. This liquid was steamed down to the volume of 1 c.c. of which $0 \cdot 2$ c.c. was injected intracutaneously into a tuberculous guineapig. The effect after 2 days was a superficial necrosis about the size of the half of a sixpence. The control (tuberculous guinea-pig) succumbed after a dose of 0.05 c.c. of pure tuberculin. These injections have been repeated several times both with tuberculous and non-tuberculous animals. In all cases the disappearance of the tuberculin from the peritoneal cavity was rapid and complete. 
The foregoing experiments show that tuberculin rapidly disappears when injected intravenously or intraperitoneally into the organism, but they give no information about its fate. Two possibilities may come into consideration. As was shown by Franceschelli (1913) the total quantity of injected tuberculin cannot be found in the urine till after the lapse of several hours. In the meantime the substance must be stored outside the vascular system, or it must be present in the blood in a combination which does not give the tuberculin reaction. Now the question is, has the blood in vitro or in vivo any power of binding tuberculin?

Experiments which were carried out to solve this question gave a definitely negative result.

By mixing and incubating extract of fresh ox-liver in salt solution with tuberculin, I found no evidence that any of it was fixed, so I proceeded to try if the living cells themselves would have this power.

Guillain and Laroche (1910) found that tuberculin was bound by brainsubstance. But according to these authors the mixture of brain and tuberculin was even more toxic than tuberculin alone. By mixing fresh paste of ox-liver and -brain with tuberculin, evidence was found that the tuberculin was bound by the living cells. By boiling it could be recovered from the new combination.

Living cells therefore may fairly be supposed to possess a faculty of binding tuberculin in vitro. The possibility remains that this reaction also takes place in vivo.

In order to trace the tuberculin in the organism I have injected tuberculin into normal guinea-pigs and rabbits. According to Franceschelli, tuberculin leaves the body through the kidneys and may be recognised in the urine some hours after the injection. This statement $I$ have been able to confirm : 2 guineapigs (weight $450 \mathrm{~g}$.) were injected with 1 c.c. of tuberculin. The 24 hours' urine was collected and used for the Pirquet reaction, which was faint, but typical. (Urine from normal guinea-pigs gave no reaction.) A similar reaction was also obtained with urine from the following 48 hours. In both cases the urine was concentrated by steaming and afterwards treated with an equal volume of pure alcohol. The precipitate which formed, was washed with alcohol and dissolved in distilled water. The test was made with this solution. Tuberculin rapidly disappears from the blood, and as it cannot be recovered from the urine until at least 3 hours have elapsed from the time of injection, I killed some animals at this time to find out if the tuberculin can be demonstrated in any of the tissues. The rabbits (weight 900 to 2500 g.) were injected intravenously with 3 c.c. of tuberculin, and the guinea-pigs (weight 300 to $450 \mathrm{~g}$.) received 2 c.c. intraperitoneally.

In the blood I was never able to find tuberculin. The examination of lungs, kidneys, spleen and the contents of the bowels gave no better results. In order to detect even traces of the substance the organs were ground and boiled with water. The filtered decoction was steamed down and pure alcohol was added. The precipitate was dissolved in water and injected into tuberculous guinea- 
pigs. Extracts from the liver, which had been prepared in this way, in three cases gave a slight necrosis after injection. In eight cases I got a distinct, though small necrosis after injection of extract from muscles and bones, and in three cases when extracts were prepared from muscles and bones separately, I found that whereas the extract from bones gave a distinct reaction, the extract of muscles had no such effect. By these injections I have, however, never been able to produce as strong a reaction as that effected by pure tuberculin. Control-injections into normal animals were always negative.

It must therefore be concluded that the reaction which takes place in vitro by the binding of tuberculin in living tissue to a certain extent may take place also in vivo, and that the binding here is mainly effected by the bones. The circumstance that tuberculin cannot be quantitatively recovered in the organism after injection, possibly means that the tuberculin now forms a new combination, which we cannot detect by our methods. This may also explain why we have not been able to find tuberculin in the tuberculous organism. Many attempts have been made to isolate tuberculin from affected organs, but always in vain. My experiments in this respect have been equally without result. Recently I have tried to isolate tuberculin from the bones of tuberculous animals and men. From lack of material I have not, however, been able to complete these experiments. - Not until we obtain certain knowledge regarding the fate of tuberculin in the organism shall we be able to determine, whether it is a true product of the tubercle bacillus or an artefact, formed by the decomposition of the real toxin.

\section{EXPERIMENTS WITH COMPLEMENT-BINDING.}

The evidence of a certain immunity in tuberculosis is not supported by facts which can give us any definite idea of its exact nature. Whereas in diphtheria and tetanus the immunity can be explained by the presence of substances which have the faculty of neutralising the toxins of these diseases, in vitro and in vivo, in tuberculosis we have not been able to demonstrate a similar antitoxin. As a means of solving this question, much attention has been paid to the complement-binding antibodies, which, however, as far as we know, play no great part in the immunology of tuberculosis. Although it has been proved that in many cases of tuberculosis in men and animals the presence of complement-binding antibodies may be demonstrated, yet where we most expect to find them, in the blood of individuals which have recovered from their disease, very often no trace of them can be found (vide Löwenstein and others).

As previously mentioned, the method of complement-binding has not given very important results in the immunology of tuberculosis. In spite of much effort we still know very little about the relation between the tuberculous antigen and its antibodies. As will be described below I have tried to throw some new light on these questions, using for the purpose a somewhat modified version of the technique, commonly applied in studies of complement-binding. 
The object of my experiments has not been to determine the quantity of complement-binding antibodies in tuberculous sera, but to investigate the nature of the alexin in these sera, and the nature of its combinations with antigen and antibodies.

The complement used in my experiments has mostly been complement from human sera. In some cases I have used complement from guinea-pigs and rabbits. Before proceeding to the main experiment the quantity of complement in the sera was always determined by titration. This was carried out by adding active serum in varying quantities to tubes containing equal volumes of washed corpuscles and inactivated rabbit's amboceptor, which was prepared by injecting a rabbit intravenously with sheep's erythrocytes. In all experiments the amboceptor was used in doses which had the power of dissolving twice the quantity of erythrocytes that was used. During the months in which the experiments were made the haemolytic power of the amboceptor remained practically unaltered. It was stored undiluted in the refrigerator. Because of the small quantities of human serum which were at my disposal, it was expedient to use a very thin emulsion of erythrocytes. In all experiments I therefore used a 0.5 per cent. emulsion. The serum was diluted 1 to 10 in all experiments. (Rabbit's serum was diluted 1 to 4 .)

In order to determine the complement-quantity of the sera, 0.5 c.c. of a 0.5 per cent. erythrocyte-emulsion and 0.003 c.c. of amboceptor from a rabbit treated intravenously with sheep's erythrocytes, were filled into 10 tubes. To all tubes was added the unheated serum, which was diluted with salt solution, in quantities varying from 0.08 to 0.28 c.c. Finally the contents of the tubes were brought to the same volume by means of salt solution, and the tubes were incubated for $\frac{3}{4}$ of an hour. The smallest quantity of diluted serum, which was sufficient to bring about a complete haemolysis I have called the titre $(\mathrm{T})$ of complement. If the haemolysis was complete in all tubes, or if it was not complete in any of them, the titration had to be repeated with smaller or greater doses of serum.

As the emulsion of erythrocytes is not quice constant from day to day, the titre found by this method is no absolute measure of the quantity of complement contained in the sera. Nevertheless we obtain a relative measure of the contents of complemen' in sera which are investigated at the same time. The difference between the absolute and the relative values of the titres can, however, not be great. This will be seen from the titres of sera examined on different occasions. Of 349 sera in which the complement was examined, 83 per cent. had a titre between $0 \cdot 10$ and $0 \cdot 20$. 25 per cent. had a titre of $0 \cdot 12$. A small number of sera, having a titre above $0 \cdot 30$, were not used for further experiments. As the blood-samples were always kept under the same conditions, and examined 2 to 24 hours after the drawing of the blood, the diagram (Fig. 1) will give us a good idea of the contents of complement in human sera. The sera belonged to all sorts of persons, old and young (not infants), sound or suffering from various maladies. 
As will be seen from Fig. 1, the amount of complement in the sera was found to be constant within certain limits. No relation could be found between the state of health of the donor of the blood and the quantity of complement in his serum.

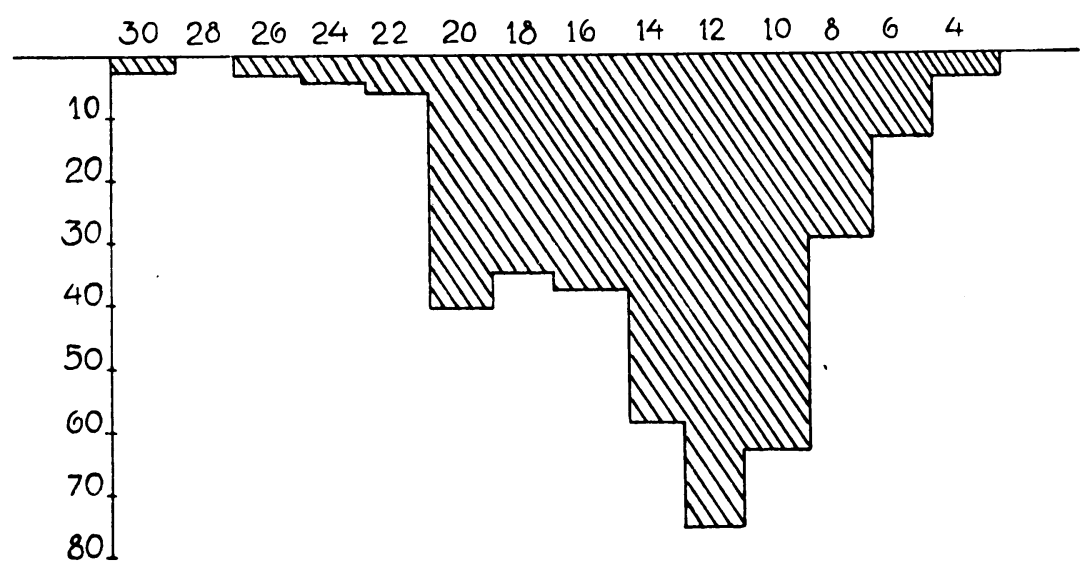

Fig. 1. Diagram showing the complement content of the sera evamined.

(Numbers along the top indicate the complement-titre, numbers along the side the numbers of sera examined.)

After the quantitative determination of the complement the main experiment was carried out, the object of which was to compare the combination or binding, which was effected between the tuberculous antigen, alexin and tuberculous amboceptor in the different sera. One experiment, accordingly, always gave me a comparison between two different sera.

Before the experiment eight tubes are arranged in two rows of four (see Fig. 2). All of the tubes receive $0 \cdot 25$ c.c. of a 10 per cent. solution of tuberculin in 0.85 per cent. salt solution. The two sera $A$ and $B$ are filled into the tubes, into the upper row of tubes active serum $A$ and inactivated serum $B$, into the lower row inactivated serum $A$ and active serum $B$. The tubes marked I contain exactly the quantity of complement which is necessary to dissolve 1 c.c. 0.5 per cent. emulsion of sheep's corpuscles. Bui whereas the complement in the upper row of tubes is represented by the complement of serum $A$, the complement in the lower row belongs to the serum $B$. The tubes, as will be seen, in all other respects have the same contents. The tubes marked II contain one and a half, the tubes marked III twice, and the tubes marked IV thrice as much complement as the tubes marked I. Finally equal volumes in all tubes are obtained by adding 0.85 per cent. salt solution. Now the tubes are placed in the incubator for ${ }_{4}^{3}$ of an hour in order to effect the binding of the complement by means of tuberculous antigen and amboceptor. After removal from the incubator 1 c.c. of erythrocyte-emulsion, with twice as much rabbit's amboceptor as is required to dissolve it, is added to all tubes, which are again 
put into the incubator for $1 \frac{1}{2}$ hours. Now the tubes are examined and the result of the haemolysis is noted.

To exclude errors arising from incomplete inactivation of the sera, controls were often made with the inactivated serum, amboceptor and corpuscles. The inactivation was always complete.

As the tubes in the two rows in couples contain exactly the same amount of antigen, amboceptor, complement and serum, the haemolysis in the two rows

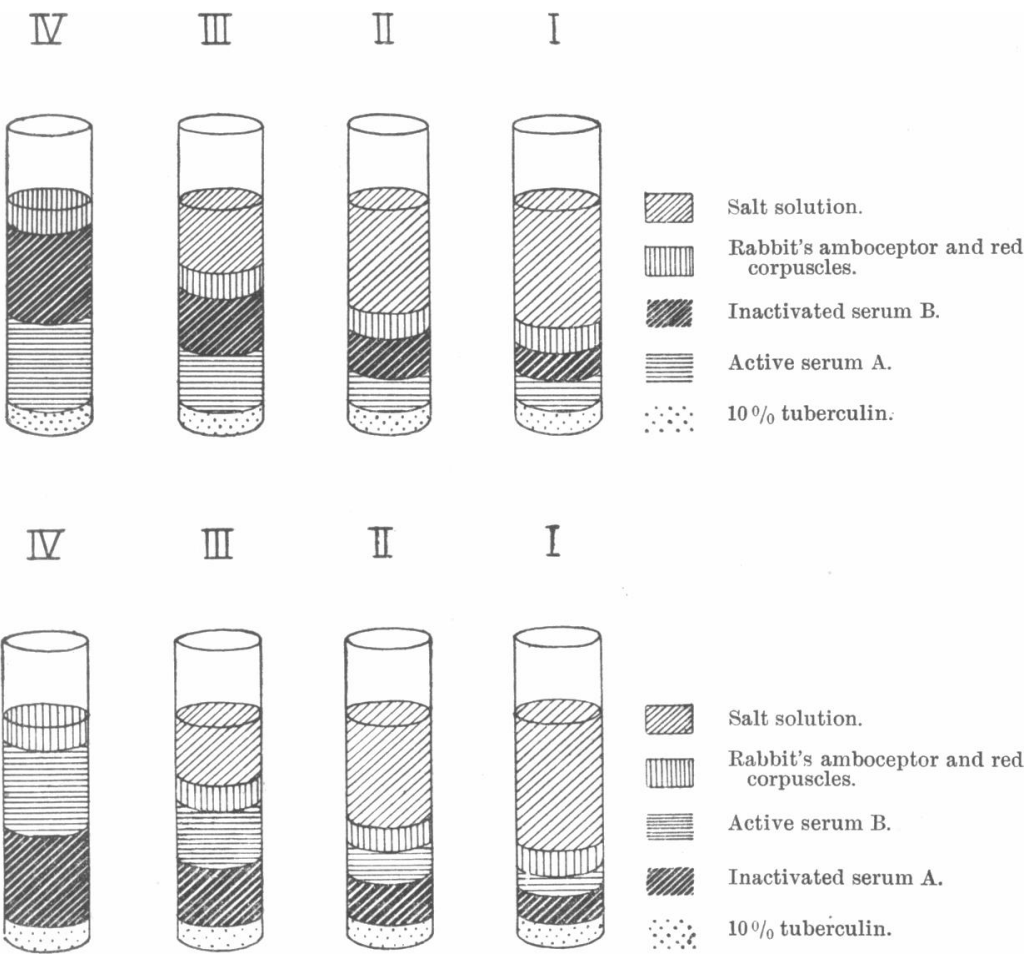

Fig. 2.

must be supposed to be parallel. This is, however, as will be seen from my experiments, not the case.

In my tables XXX means total, and XXO partial haemolysis. XOO indicates traces of haemolysis and 000 no haemolysis.

As the contents of the tubes only differ in regard to the quantities of sera, in my tables I have only written down the complement-titre (T) of the sera, and the results of the haemolysis. The tubes marked II always contain one and a half, the tubes marked III twice and the tubes marked IV thrice as much complement as the tubes marked I.

From the following experiments it will be seen that the haemolysis in the two rows is not parallel although the amounts of complement in the two rows are equal. Similar results which were obtained through numerous experiments, show us that some complements will be more readily fixed than others. 
Expt. 1. (17. x. 1918.)

Abbreviations: $\mathrm{S}=$ serum. $\mathrm{R}=$ rabbit. $\mathrm{M}=$ man. $\mathrm{G}=$ guinea-pig. $\mathrm{T}=$ titre. $\mathrm{H}=$ haemolysis. Tu $=$ tube. U.r. $=$ upper row of tubes. L.r. =lower row of tubes. $\mathbf{A}=$ active. Ina=inactivated.

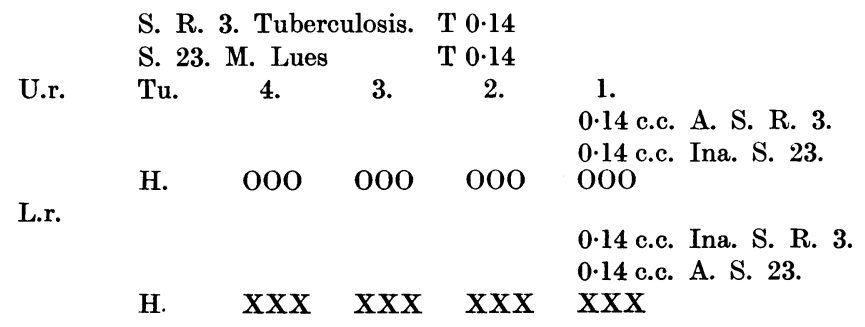

Expt. 2. (17. x. 1918.)

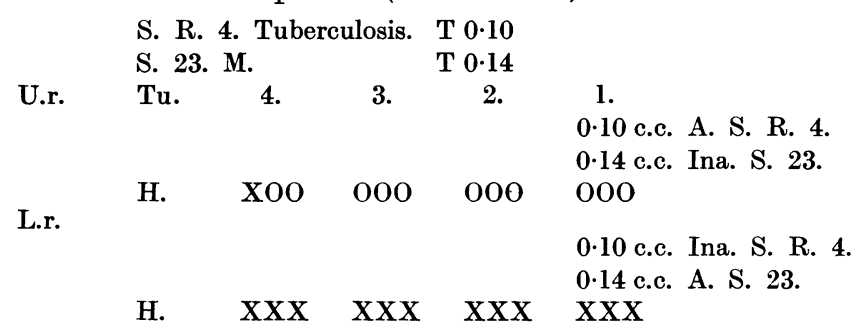

This phenomenon, in my opinion, can only be interpreted in one way. What is called complement or alexin is not the identical substance in all sera, thus the complement in rabbit's serum differs constitutionally from that in human serum.

The view that there are a plurality of complements was first advanced by Ehrlich and Morgenroth (vide Ehrlich, 1900), who demonstrated several facts which strongly supported it. They stated that "experimental results positively show a multiplicity of complements in normal serum." This hypothesis has, however, been vehemently opposed by many authors, especially by Buchner and Bordet (vide Bordet, 1920), who maintain the existence of a single complement. As will be seen, my observations are in full accordance with Ehrlich's view.

The difference which I could demonstrate between the binding of rabbit's complement and human complement can, as the following experiment will show, also be demonstrated between other heterologous sera, for instance, between the complement of rabbit and guinea-pig.

Expt. 3. (16. xi. 1918.)

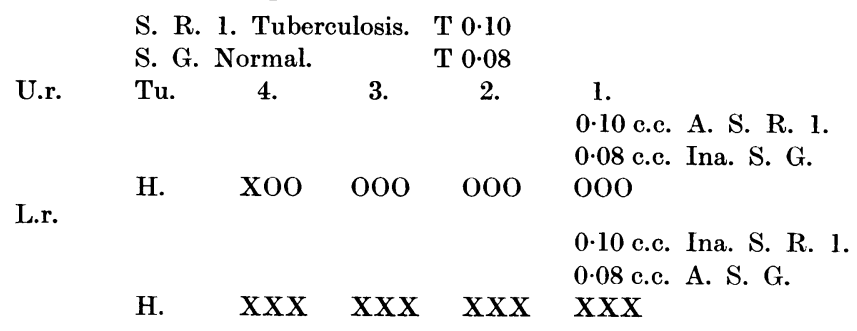


The complement of 10 guinea-pigs, compared with the complement of 13 human sera in four cases showed some difference.

As will be seen from the experiments which are mentioned above, the comparisons in all cases have been made between heterologous sera. Now the question is, can a similar difference be demonstrated also between homologous sera? The following experiment answers the question affirmatively.

$$
\begin{aligned}
& \text { Expt. 9. (29. xi. 1918.) }
\end{aligned}
$$

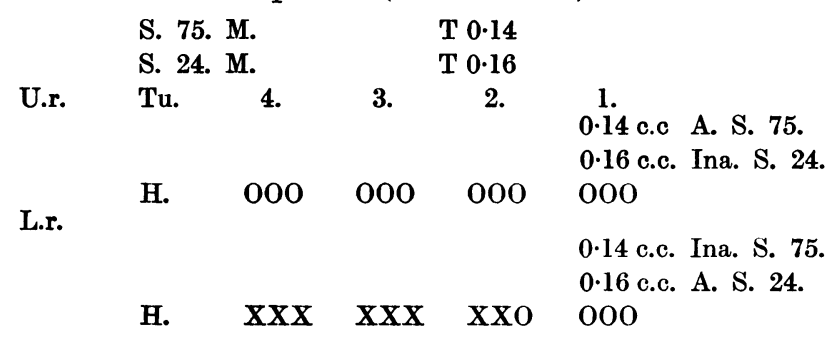

The difference which I have been able to demonstrate between the complements may be ascribed theoretically to the circumstance that one complement is able to make combinations which other complements cannot. These combinations may arise either between specific tuberculous components of the antigen (tuberculin) and the corresponding amboceptors, or between nonspecific components and corresponding "accidental" amboceptors in the sera.

In the case of human sera the existence of tuberculous amboceptors is very difficult to exclude, because of the frequent and often unobserved tubercular infections in man. Very often buman sera must be supposed to contain both tuberculous and other amboceptors, which are able to unite with nonspecific components of tuberculin. In the case of laboratory animals, however, we know to a certain degree the factors with which we have to deal.

Below I will give the results obtained in experiments with homologous sera from normal and tuberculous laboratory animals.

On examining the sera of eight normal rabbits with the technique which I have described, no difference could be found between the complements. But the complements of these eight sera and five other sera, also from normal rabbits, in most cases showed a marked difference from the complements of sera from four tuberculous rabbits.

$$
\begin{aligned}
& \text { Expt. 10. (26. x. 1918.) } \\
& \text { S. R. Tuberculosis. T } 0 \cdot 14 \\
& \text { S. R. 20. Normal. T } 0 \cdot 14
\end{aligned}
$$

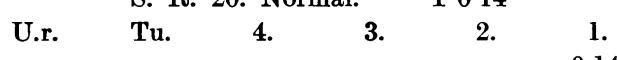

$$
\begin{aligned}
& \text { 0.14 c.c. A. S. R. Tuberculosis. } \\
& \text { 0.14 c.c. Ina. S. R. } 20 . \\
& \text { L.r. H. XOO } 000 \quad 000 \quad 000 \\
& 0 \cdot 14 \text { c.c. Ina. S. R. Tuberculosis. } \\
& \text { H. } \quad \mathrm{XXX} \quad \mathrm{XXO} 000 \quad 000
\end{aligned}
$$


In most cases where a difference could be demonstrated the tuberculous complement was more completely bound than the normal complement. In four cases no difference could be shown between the two complements.

In one experiment a comparison was made between the complements of a tuberculous and a normal guinea-pig, but no difference could be demonstrated.

The experiments with human sera may be divided into two groups, (1) those with complement derived from persons whose relation to tuberculosis was unknown, (2) those with complement derived from tuberculous persons.

(1) Experiments with complements from persons who have not suffered from tuberculosis or whose relation to previous tubercular infection is unknown.

Of this category 182 sera were examined. Twelve of these gave a different reaction to the others.

(2) Experiments with complements from persons suffering or having previously suffered from tuberculosis.

Thirty-five sera from tuberculous persons were examined. They were compared with 296 sera from other persons, the majority of which were the sera mentioned under (1).

These experiments show $(a)$ that the complements of different sera not infrequently show differences; the complement of a guinea-pig is not identical with that of a rabbit or of a man; (b) that differences also may be found between complements derived from individuals of one species; $(c)$ that probably the complement of a tuberculous individual differs from that of the healthy subject. Of 182 complements belonging to persons who had not suffered from tuberculosis, or who were not known to have suffered from tuberculosis, only 12 differed from the others. Thirty-five tuberculous sera showed a distinct difference when compared with 207 sera from non-tuberculous individuals, whereas no difference could be demonstrated in 86 cases. In some cases the haemolysis was more complete in the tubes containing tuberculous complement, in other cases it was more complete in the tubes with non-tuberculous complement. As will be seen from the experiments, however, in many cases a striking conformity could be observed and the bindings of tuberculous complements in many cases were absolutely parallel.

I am fully aware of the circumstance that what I have called non-tuberculous complements very often belong to individuals who must be regarded as infected with tuberculosis. This circumstance may, perhaps, account for the cases where a difference between tuberculous and other complements could not be demonstrated. My object has only been to demonstrate a difference between complements of persons who have been subjected to grave tuberculous infections and complements of persons who have not been subjected to tuberculous infections, or only to infections which may be regarded as inconsiderable.

Against the technique which I have used in this work certain objections can be made.

Moro (1907) stated that the titration of human complement is not exact

Journ. of Hyg. xx 
when performed with the technique which I have described above. In some cases a better result may undoubtedly be obtained by Moro's method, but even if the titration is carried out in the way which is described by him, we cannot be certain of finding the exact amount of complement contained in the sera. In most cases I believe that the ordinary technique will serve its purpose as well as any other. This opinion is based upon the following facts.

1. The amounts of complement which $I$ have found in human sera are on the whole the same as were found by Moro.

2. A great many of my sera, after titration, were mixed with amboceptors from other sera and the complement was titrated repeatedly. (The main experiment is really a titration of the complement.) If the first titration had given a wrong result owing to a lack of human amboceptor, later titrations with addition of different amboceptors never could give the same result in a series of experiments. This was, however, very often the case.

3. In the titration of complement from rabbits and guinea-pigs Moro has no objections against the ordinary technique. These complements, however, showed the same differences as human complements.

As was observed by Fridemann (1910) and others, a normal serum may give a positive Wassermann reaction, whereas this reaction disappears after inactivation. Fridemann has proved that the phenomenon depends upon the globulin-content of the serum, but he also states that it is of no practical importance where sera from men and guinea-pigs are concerned. The difference which I have found between the complements accordingly cannot be explained as an analogy to this phenomenon. And in no case can it explain the difference existing between tuberculous and non-tuberculous complement. In some experiments I have made a control experiment which fully proves that my results have nothing to do either with a deficient method of titration or with the phenomenon observed by Fridemann. One of these control experiments will be related below:

\begin{tabular}{|c|c|c|c|c|c|c|}
\hline \multirow[b]{2}{*}{ U.r. } & \multicolumn{2}{|c|}{ S. R. Normal. } & & T. O. 14. & \multirow[b]{2}{*}{ I } & \multirow{3}{*}{$\begin{array}{l}0 \cdot 20 \text { c.c. } \text { S. G. A. } \\
0 \cdot 14 \text { c.c. } \text { S. } \text { R. Ina }\end{array}$} \\
\hline & Tu. & 4. & 3. & 2. & & \\
\hline \multirow{4}{*}{ L.r. } & & & & & & \\
\hline & H. & OOO & 000 & 000 & 000 & \\
\hline & & & & & & 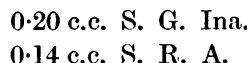 \\
\hline & H. & $\mathbf{X x X}$ & $\mathbf{X X X}$ & $\mathrm{x} \cap 0$ & 000 & \\
\hline
\end{tabular}

The control experiment was performed without the addition of tuberculin, which was used in the main experiment. Whereas the haemolysis in the latter was as related above, in the control the haemolysis was complete in all tubes. This experiment shows beyond a doubt that the complement-binding by the combination between tuberculin and corresponding amboceptors has occasioned the different results of the haemolysis in the two rows of tubes. 


\section{Experiments with phagocytosis.}

Summing up the results obtained by innumerable workers with the general and known methods of immunological investigation, one has to admit that the riddle of immunity in tuberculosis is still unsolved, and, bearing this in mind, attempts to solve the problem must necessarily be based upon new methods. I have therefore adopted the methods of Wright for the examination of leucocytes, hoping in them to find a finer indicator of the effects of the tuberculous agents than is represented by the whole organism of men and animals.

The object of my work has been to determine whether tuberculin, the substance which is supposed to be the toxin of the tubercle bacillus, has any toxic effects upon the leucocytes, and whether it affects the leucocytes from a healthy organism in the same way as it affects those from an organism suffering from tuberculosis.

One of the vital functions of the leucocytes is their motility. Does tuberculin affect this motility? In order to study this question I have used the method of the emigration or chemotactic tubes of Wright (1915).

The emigration tube was first filled to midway point with blood from a puncture in the finger and tuberculin added by superimposing it upon the unclotted blood.

When undiluted tuberculin was superimposed upon my blood no emigration of the leucocytes into the white clot took place. This inhibitory effect of the tuberculin on the wandering of leucocytes may be thought to be caused by osmotic conditions in the fluids above the red clot. I found that a 0.5 per cent. salt solution has a lower, and a 1.5 per cent. salt solution a higher molecular tension than tuberculin. As both these concentrations, however, when superimposed upon the blood, effected a marked emigration of leucocytes into the white clot it is obvious that molecular conditions cannot be the cause of the inhibitory effects of tuberculin. As we know, tuberculin consists of glycerinbroth and a decoction of bacilli, and it is therefore possible that the glycerin or the broth or both of them may inhibit the emigration. A 10 per cent. solution of glycerin in broth was steamed down to the consistence of tuberculin, and superimposed upon the blood. This fluid inhibited the emigration of the leucocytes to some extent, but when diluted 1 to 5 with salt solution emigration was abundant. Tuberculin, on the other hand, even when diluted 1 to 5 and 1 to 25 with salt solution, totally inhibited the emigration, whereas a dilution 1 to 50 had no such effect. These experiments clearly show that tuberculin has a distinct and characteristic inhibitory effect upon the motility of leucocytes. The white corpuscles are similarly affected also by tetano-toxin and diphtheria-toxin. Vaillard (1891) demonstrated that tetano-toxin has a negative chemotactic influence upon leucocytes in vivo. It is easily demonstrated in vitro both for tetano-toxin and diphtheria-toxin. If tetano-toxin, undiluted or diluted 1 to 5 , or diphtheria-toxin, undiluted or diluted 1 to 25 , 
is superimposed upon blood in the way described, no emigration of leucocytes will take place. This toxic effect can, however, be neutralised by antitoxins. Undiluted horse-serum has an inhibitory effect upon the motility of human leucocytes, but when it is diluted and added to toxin, a great number of them will wander into the white clot, if the mixture is superimposed upon blood. The leucocytes in this way give us an indicator of the action of toxin and antitoxin. It may therefore be thought that similar phenomena may be demonstrated also under different circumstances, for instance, where the tuberculous toxin comes into contact with the unknown tuberculous antitoxin. In order to test this hypothesis I incubated mixtures of tuberculin with serum from persons suffering from tuberculosis, and from persons who have conquered the infection, and whose organism accordingly may be supposed to contain antitoxin. The inhibitory effect of tuberculin on the motility of the leucocytes could, however, in no case be neutralised.

The results obtained with the emigration tubes clearly show that tuberculin has an effect upon white blood corpuscles, and that it cannot be regarded as an indifferent substance. In my experiments I have mostly used my own blood as test object, and because I give a positive Pirquet reaction, my blood may be regarded as tuberculous blood, and the effect of tuberculin upon my leucocytes therefore be explained as the effect upon tuberculous cells. This objection is, however, of no value, because leucocytes from normal rabbits and guinea-pigs are affected in quite the same way by tuberculin.

The emigration tubes cannot give us a quantitative measure of the effects of tuberculin upon white corpuscles. In order to obtain this I used the opsonic technique of Wright (1912).

For my purpose I used blood from my finger, and, having washed and centrifuged the corpuscles in the ordinary way they were divided into two equal parts. One of these parts was mixed with tuberculin, and the other with 0.85 per cent. salt solution. Then the tubes were incubated for one hour at $37^{\circ} \mathrm{C}$. After this time the tubes were centrifuged and the corpuscles washed three times with salt solution. Samples from both tubes were mixed with the same emulsion of staphylococci and the same serum according to the ordinary technique. The cytophagic index was determined by counting the number of staphylococci which were enclosed in at least 50 leucocytes. By this method one is able to obtain a quantitative measure of the effect of tuberculin upon another of the vital functions of the leucocytes, the phagocytosis.

These experiments showed that tuberculin had a distinct inhibitory influence upon the phagocytosis. Controls proved that this effect was not caused by the contents of glycerine and salts of the tuberculin. The same effect was demonstrated in the tuberculin which had been purified in the way described by Koch (1912, vol. I, p. 674).

The picture which we obtain when staining after having treated the leucocytes with toxin is quite different from the normal. Whereas the latter shows us the leucocytes beautifully stained and enclosing the staphylococci, 
in the former the phagocytosis, if present, is decidedly smaller, and the leucocytes are badly stained with indistinct differentiation between protoplasm and nucleus.

In order to evade those constituents of tuberculin which come from the glycerin broth, I have cultivated tubercle bacilli on a solid medium, and afterwards boiled them in sterile, distilled water. After filtering the decoction it was steamed down to dryness at a temperature of $56^{\circ} \mathrm{C}$., and dissolved in 0.85 per cent. salt solution. With this solution, which caused no trace of haemolysis, I have treated the leucocytes, using the same technique as before. Whereas white blood corpuscles which were kept for 2 hours in the incubator at $37^{\circ} \mathrm{C}$., and afterwards mixed with staphylococci and serum, on an average contained nine staphylococci (50 leucocytes and 450 staphylococci counted), those corpuscles which were treated with decoction of tubercle bacilli instead of salt solution, were highly degenerated and contained no staphylococci at all.

These experiments leave no doubt as to the toxic effect of tuberculin on leucocytes and phagocytosis. But about the nature of this effect we know very little. On the one hand it is possible that tuberculin has a similar effect upon the cells as other bacterial toxins (i.e. diphtheria toxin), on the other hand there are many who hold that the effects of tuberculin upon the organism must be ascribed to anaphylaxis.

As has been demonstrated by Gengou (1898), diphtheria- and tetano-toxin have no effect upon monocellular organisms, and Bordet (1896) found that a contact of 3 to 4 hours with diphtheria-toxin did not diminish the phagocytic power of leucocytes. My experiments serve but to confirm the results of Bordet.

Working error: In my opsonic work 1 have estimated my working error at 10 per cent. On counting a great number of control films the results never varied more than 10 per cent. My technique has been very time-consuming, a circumstance which has greatly hampered the taking of controls. On counting these I find that they represent about 15 per cent. of the total number of experiments. In fact the experiments which were made to illuminate the same question control themselves.

As previously mentioned, tuberculin cannot be regarded as a genuine toxin like, for instance, diphtheria-toxin. Now the question is, can the toxic influence of tuberculin upon phagocytosis be ascribed to anaphylaxis?

What we know about the nature of anaphylaxis is that it will manifest itself in a number of quite characteristic clinical symptoms from the nervous, cutaneous, respiratory, vascular, alimentary and the genito-urinary systems, in short from all parts of the body. We know, furthermore, that these symptoms can be reproduced in isolated organs, as for instance in the isolated heart or uterus, and that the state of hypersensitiveness is due to an altered condition of the blood. As an explanation of these phenomena it is supposed by many that the noxious agent which is the cause of the anaphylactic shock has its point of attack in the nervous system (Besredka, 1911). Sufficient experiments have not been carried out to determine whether the anaphylactic 
reaction is a universal reaction or not. This possibility must, however, be taken into consideration. In fact some of the manifestations of hypersensitiveness can more easily be explained as a cellular reaction, caused by the influence of the noxious agent upon the so-called sessile receptors in the cells. As mentioned, anaphylactic symptoms can be reproduced in isolated organs. Arnoldi and Leschke (1920) demonstrated anaphylactic reactions in organs which have been completely freed from all traces of blood. The local reaction which is seen as the result of an injection of serum into the skin of animals previously treated with the same serum, although generally accepted as a symptom of anaphylaxis, has no resemblance to the anaphylactic shock, is not necessarily accompanied by symptoms of general hypersensitiveness, and can more easily be explained as a local cellular reaction than as the local result of the influence of the noxious agent upon the nervous system. An experiment which I have carried out points to the conclusion that the reaction may be quite independent of the nervous system. Six to seven days after therapeutic injections of horse-serum, patients were injected with a mixture of horse-serum and novocain-adrenalin which effected a complete anaesthesia. The local reaction was always typical and of the same dimensions as the controls.- Summing up what is really known about anaphylaxis one must therefore admit the possibility of a cellular hypersensitiveness, which may manifest itself with a cellular anaphylactic shock.

In order to investigate this question and in continuance of the experiments described above, I have attempted to produce anaphylaxis in cells from sensitised organisms. As I wanted to work with isolated cells which were capable of living outside of the organism, I have used leucocytes as my testobject, the vital functions of which may be partially controlled by the technique which I have described.

Numerous experiments demonstrated that:

(a) In experimental anaphylaxis the cytophagic index was unaltered before and after the shock, in tuberculous guinea-pigs before and after tuberculin-injections.

(b) The anaphyla-toxin (Friedberger, 1917) does not always affect the cytophagic power of the leucocytes, although in some cases of serum-disease an inhibitory effect of horse-serum upon leucocytes was demonstrable.

If we mix tuberculin with serum from a tuberculous or a normal animal, afterwards exposing leucocytes to the effects of this mixture, we will find that the noxious influence of the tuberculin upon the phagocytosis has been partly neutralised by the serum. This is demonstrated by the following experiment.

Blood from a normal guinea-pig was treated for $1 \frac{1}{2}$ hours at $37^{\circ} \mathrm{C}$. with:

(a) Salt solution. Cytophagic index, 5•8. (289 staphylococci and 50 leucocytes counted.)

(b) Tuberculin, diluted 1 to 10 with salt solution. Cytophagic index, 0.98. (49 staphylococci and 50 leucocytes counted.)

(c) Tuberculin, diluted with one volume of serum from a tuberculous 
guinea-pig and eight volumes of salt solution. Cytophagic index, 2.66. (133 staphylococci and 50 leucocytes counted.)

(d) Tuberculin, diluted with one volume of serum from a normal guineapig, and eight volumes of salt solution. Cytophagic index, 5·18. (258 staphylococci and 50 leucocytes counted.)

The protective power of the serum against the noxious influence of the tuberculin upon the phagocytosis is not removed by the destruction of the complement. Serum from normal animals practically always had a stronger protective power than serum from tuberculous animals. It was, however, not possible to ascertain whether the tuberculous leucocytes were more sensitive to the toxic effects of tuberculin than the normal ones. This is undoubtedly due to a deficiency in the technique. In many cases the films will contain leucocytes which are so altered by the tuberculin that they are unrecognisable. This naturally causes grave errors in the results, and I have therefore not taken the trouble to continue my attempts to solve the question in this way. The toxic influence of tuberculin upon leucocytes can, however, be demonstrated directly in another way by means of the paraffin cells of Wright (1918).

Into one of these cells, with living leucocytes, we put a drop of tuberculin, diluted 1 to 20 or 1 to 100 and neutralised with sodium hydrate. Into a control cell we put a drop of salt solution and place the slide in the moist chamber at $37^{\circ} \mathrm{C}$. for $1 \frac{1}{2}$ hours. The slide is again withdrawn and the tuberculin is washed off with salt solution. This done, we fix with corrosive sublimate and stain with methylene blue. In the cells where the tuberculin has been a number of the leucocytes have disappeared, and a great number of the remaining leucocytes will present a picture of degeneration. The protoplasm is shrunken and the differentiation between protoplasm and nucleus is indistinct. In some places we find badly stained heaps, which must be regarded as the remnants of degenerated leucocytes.

These methods will, however, not give us a quantitative means of measuring the effect of tuberculin upon normal and tuberculous leucocytes. This may be obtained in another way, which I will describe below.

An injection of pure tuberculin into the peritoneal cavity of a guinea-pig will produce a rich exudation of leucocytes into the peritoneal fluid. If injections are made at the same time into tuberculous and normal guinea-pigs, the exudates will be seen to differ in several respects. Whereas the exudate from the normal guinea-pig will coagulate slowly, the exudate from the tuberculous animal will coagulate within a few minutes. Also the leucocytes of the two exudates are different. This difference can be demonstrated by vital staining. The exudates are centrifuged and washed with salt solution. The leucocytes are mixed with a drop of Manson's stain and placed upon a glass slide under a covering glass, which is fixed to the slide with vaseline or melted paraffin. The main difference in the staining of the two sorts of leucocytes consists in the staining of granules, which, in the normal leucocytes, are beautifully stained red, and are present in such numbers as to render 
the nucleus almost invisible. In the tuberculous leucocytes these granules have disappeared, and the nucleus is very distinctly seen, surrounded by unstained protoplasm. The difference can also be demonstrated by an addition of aqueous neutral-red to the leucocytes. In the normal leucocytes the granules stain red, whereas the tuberculous leucocytes have no granules. This difference is caused by a different reaction of the protoplasm in the leucocytes. The reaction is normally slightly acid. In the tuberculous leucocytes this acidity is diminished. This difference in the mode of vital staining is identical with that which is observed in the staining of living and dead leucocytes. If normal leucocytes and leucocytes which have been heated above the flame are mixed with Manson's stain quite the same differences will be seen. In living leucocytes the granules are stained, in heated leucocytes no granules will be seen. Leucocytes from the tuberculous animal must be considered as dead or dying and it must therefore be concluded that leucocytes from tuberculous organisms are more sensitive with regard to tuberculin than those from a normal organism. My experiments in this respect confirm the observation of Achard and Bénard (1909), who employed a technique which is unknown to me but state that "l'activité leucocytaire" in a solution of tuberculin is not so good in leucocytes from a tuberculous organism as in normal leucocytes.

The phenomenon which I have described above is constantly observed in the leucocytes of tuberculous guinea-pigs. I have been able to demonstrate it in eight out of nine tuberculous animals. Most striking were the changes where the tuberculosis was far advanced. Control animals (10) never showed the phenomenon, which must be regarded as a cellular allergic reaction, the tuberculin reaction of the cell.

\section{SUMMARY.}

In order to obtain knowledge of the fate of tuberculin in the organism the substance was injected into normal animals. After injection, tuberculin rapidly disappears from the vascular system and does not reappear in the urine till after several hours. In the meantime the substance is bound in the organism, probably in the bones and in the liver. Living cells in vitro also have the faculty of binding tuberculin.

Through numerous experiments a difference is demonstrated between the complements of different sera, a fact which strongly supports the theory of the plurality of complements. Evidence is brought forth which points to a difference between tuberculous and non-tuberculous complement.

Tuberculin is highly noxious to the vitality of the white blood corpuscles, and more toxic to corpuscles from tuberculous organisms than from nottuberculous organisms. A certain protective power in regard to the toxic action of tuberculin is found in the serum. This power is greater in serum from normal organisms, than in serum from tuberculous organisms.

By means of vital staining a difference is demonstrated between the effects of tuberculin upon the leucocytes from normal and tuberculous animals. 


\section{REFERENCES.}

Achard et BÉnard (1909). Compt. Rend. Soc. Biol. Lxvit. p. 502.

Arnoldi, W. und Leschke, E. (1920). Deutsche med. Wochenschr. No. 37.

Behring (1912). Cited in Dieudonné's Schutzimpfung und Serumtherapie, 2nd ed.

Besrejka, Kraus und Levaditi (1911). Handbuch der Technik und Methodik der Immunitätsforschung. Ergänzungsband. (Jena.)

Bordet, J. (1896). Ann. Inst. Pasteur, x. p. 110.

- (1920). Traité de l'Immunité, p. 447. (Paris.)

EHRLich, P. (1900). Berlin. klin. Wochenschr. No. 31.

Franceschelli (1913). Zeitschr. f. Immunitätsforch. Orig. xx.

Fridemann (1910). Zeitschr.f. Hyg. Lxvil. p. 279.

Friedberger (1917). In Kraus und Brugsch's Spezielle Pathologie und T'herapie innerer Krankheiten, II.

Gengou (1898). Ann. Inst. Pasteur, xiI. p. 465.

Guillain et Laroche (1910). Compt. Rend. Soc. Biol. LxxviII. p. 220.

Косн, R. (1912). Gesammelte. Werke, I (Leipzig.)

Löwenstein (1908). In Kraus-Levaditi's Handb. d. Tech. u. Method.d. Immitätsforsch.p. 826. (1908). In Kolle-Wassermann's Handb. d. pathog. Mikroorg. 2nd ed. v. p. 608.

Marie et Tiffman (1900). Compt. Rend. Soc. Biol. Lxiv.

Moro, E. (1907). München med. Wochenschr. Nos. 21, 31.

RUPPEL und RickMANN (1910). Zeitschr. f. Immunitätsforsch. vi. p. 34.

Slatinéanou et Danielopol (1908). Compt. Rend. Soc. Biol. lxiv. p. 420.

Slatinéanou et Jonesco-Mihaesti (1908). Ibid. p. 420.

VAILlard (1891). Ann. Inst. Pasteur, v. p. 1.

Wolff-Eisner (1909). Centralbl. f. Bakteriol. Abt. l, Referate xxxiv. p. 114.

WRIGHT, Sir A. (1912). Technique of the Teat and Capillary Glass Tube. (London.)

- (1915). Proc. Roy. Soc. Med. viII. p. 41.

- (I. 1918). Lancet, I. 